\title{
How to evaluate a graduate studentship, or choosing the right doctoral advisor
}

\author{
Duncan T Odom*
}

A potential graduate student may spend more time in deciding what trendy digital device to buy than the environment and conditions of a studentship that guide their future career - I know this is true, because I got a really good Walkman, back in the day. This brief essay summarizes the core advice that I give folks who are about to take the first steps on the (potentially perilous) path towards an academic or industrial research career in biological sciences.

\section{The golden rules}

This article is written for someone who is passionate about science, and who knows that having a doctorate is the first magical step toward a principal investigator position in a research institute or a university. You have made all the right moves so far in your undergraduate studies; so you have (a) solid to excellent grades, and (b) done substantial amounts of research with a faculty member who can vouch for your competence (this means by the way, ideally more than a year). Now, famous people in storied places as diverse as MIT, Oxford and Singapore have invited you to interview for PhD studentships, which means you have been favorably evaluated by these programs. Congratulations!

But...how do you evaluate them: that is, the group leaders whose studentships are offered to you? In other words, how do you maximize the chances of surviving the next five (all too often brutal) years of your professional life?

My advice is based on my own experiences as a graduate student in chemistry (at Caltech, 5 years), a postdoctoral fellow in genomics (at the Whitehead and MIT, 6 years), and a faculty member in comparative and functional genomics (at University of Cambridge, 6 years). I currently run a medium-sized research group with a steady state of two to four postdocs, two to three graduate students, and about four staff scientists of

*Correspondence: Duncan.Odom@cruk.cam.ac.uk

University of Cambridge, Cancer Research UK - Cambridge Institute, Cambridge, CB2 ORE, UK various flavors. Here, I emphasize points relevant to biological sciences, but some of the ideas are applicable more widely.

To begin, let's ask a quick, basic, rarely-asked question. What do you need to accomplish as a graduate student in order to get a great postdoctoral position, from which you could launch a high-caliber faculty job? Although many accomplishments might be desirable, the most basic is this:

You must obtain your doctoral degree in a reasonable amount of time (think: ideally less than five years) with (1) an intact, enthusiastic spirit of curiosity, unbroken by terrible experiences, and (2) at least one solid, firstauthor publication that has been peer-reviewed.

This clear, unambiguous goal for survival should really help guide your choice of graduate advisor with open and critical eyes. Working in a Nobel-winning laboratory and producing publications in the highest impact journals might be ideal, but might not be a realistic (or even desirable) strategy for surviving your first years as a young scientist. Since the biggest variable you can control for is the scientific identity and character of your $\mathrm{PhD}$ advisor, you should evaluate this before you join a laboratory.

\section{Established laboratories}

The easiest situation to evaluate is the established laboratory, where you should start by asking hard-nosed questions about their prior record, both in general, and specifically with graduate students' training.

\section{History with students}

How many people have accepted PhD studentships in the past?

Did they all publish first-author papers somewhere?

If not, who didn't publish, and what fraction of accepted students does that represent?

How many students have left the group, successfully and otherwise?

Where did they go?

Why?

All of these questions assume that your experience will mirror that of a typical person in that lab. 


\section{The lab's scientific leadership}

How is the advisor as a leader and scientific role model?

An interview is your chance to ask pointed questions, probably in private, with as many folks as possible. Consider soliciting gossip, either from your social network or from other labs that neighbor your prospective host lab. This advice may be particularly uncomfortable to follow, since we all want to assume the best of everyone.

But think about it this way: if you were about to adventure-drive across the Sahara desert, would you just buy any random vehicle on the word of the owner, or would you have an independent car mechanic in to evaluate its mechanical condition, and thus to maximize your survival? The first four to five years as a scientist in large part shapes one's future identity irrevocably. Again, the single biggest influence will be your advisor, so choose wisely and be as informed as possible.

To state the obvious, certain character traits are undesirable in a mentor (well, at least when present in excess). These include, but are not limited to: conflict avoidance, being a bully, having an unstable temper, overt narcissism, passive-aggressive patterns, un-professionalism (such as outright intoxication at an institute beer hour or multiple paper retractions), crippling insecurity, excessive perfectionism (never submitting papers, for example), a damaging history of playing favorites.

Use your common sense, and always remember that you are trying to avoid only serious, Titanic-sinking phenotypes. You should not be demanding perfection as everyone is human and we all have limitations and make mistakes - just look for survivability.

How much does your candidate lab's group leader travel?

Ask the laboratory if the group leader's travel to conferences and talks interferes with the lab's smooth function and publication rate. When my travel gets heavy, papers and grants turn around more slowly - and so my lab suffers. During trips, work time is more fragmented and less gets done, even when I set aside time. And, even in this wired world, I am simply much more effective in my own office, where my folks can just drop in.

\section{Lab dynamics}

How big is the prospective lab?

Having now run a group for a few years, I know from hard experience that I can only manage about five to six researchers, who are themselves actively running research projects. My realization of this limitation has meant that my lab naturally equilibrates at about ten people, counting the four staff scientists who do not normally run larger projects. If my lab got bigger than it currently is, then I would be forced to neglect people's hard-fought discoveries. My caveat here is that some faculty are much better at managing folks than I am, which leads to the next point.

Are the lab's publications spread (somewhat) evenly across all the lab members, or do publications concentrate for excessively long stretches with one or two people as first authors?

For your own sake, you should be ruthless in your evaluations, but, to be fair, do consider lab size carefully. If someone has five graduate students, and they publish three solid papers a year with different lead authors, that sounds like a potentially promising environment (averaging one paper per person, every year and a half or so). Instead, though, what if there are a total of fifteen postdocs and grad students in a single lab, and two solid papers a year get published? That situation would mean one paper published per person, every seven and a half years or so. Not quite so good.

\section{Fresh-faced group leaders}

With a newly minted junior group leader, you probably have less actual history to go on. However, you can certainly ask them questions about what kind of lab they intend to build.

How many people do they want/intend to have in the lab in five years? In what roles? Why?

Most senior postdocs do not think strongly about these issues, as most of their efforts are spent in trying to get a faculty position. However, the starting group leaders who have thought these issues through would be expected to be more prepared to train promising graduate students like you. There are powerful advantages of starting work for a very junior group, not least that your interests and theirs are exactly aligned in ways that senior group leaders cannot be. In particular, untenured group leaders will be wanting your first stories published at least as much as you do, possibly more.

An aside: I believe that the first four or five people who join a nascent laboratory have the best chances of hitting a scientific gold mine. It does, however, require courage to take a chance on an unknown group leader.

\section{Medics}

With a medically trained group leader, you have a huge advantage in easier access to patient materials, which facilitates looking at questions directly relating to human disease biology. But there are certain drawbacks.

What is the allocation of the clinician-scientist's time between clinic and lab?

Many clinician scientists stay engaged with patient care, which is often an excellent strategy, but the cost of this dedication can at times be how much attention they can give their laboratory. Furthermore, many areas of translational medicine are scientifically over-subscribed and extraordinarily competitive. Remember that as long 
as you, as a grad student, get your solid publication or two, you are fine. However, if the clinician-scientist group leader is aiming for a big splash in a top-tier journal, then lower-impact stories that make up the bedrock of a successful graduate studentship might get neglected along the way.

And finally...

Good luck!
Published: 29 April 2013

doi:10.1186/gb-2013-14-4-114

Cite this article as: Odom DT: How to evaluate a graduate studentship, or choosing the right doctoral advisor. Genome Biology 2013, 14:114. 